\title{
O Pensamento Computacional por meio da Robótica no Ensino Básico - Uma Revisão Sistemática*
}

\author{
Christiano Avila, Simone Cavalheiro, Adriana Bordini, \\ Mônica Marques
}

\author{
${ }^{1}$ Centro de Desenvolvimento Tecnológico \\ Programa de Pós-Graduação em Computação \\ Universidade Federal de Pelotas (UFPel) \\ Rua Gomes Carneiro, 1 - 96.010-610 - Pelotas - RS - Brasil \\ \{christianoavila, simone.costa, abordini,moniicamarquees\}@gmail.com
}

\begin{abstract}
The purpose of this paper is to present the state of the art in relation to the use of educational robotics as a strategy for the development of computational thinking (CT) skills for young people at the age of elementary school. For that, the methodology of Systematic Review of Literature was applied to organize and execute the revision as well as to present the results. They show that most of the studies aim to develop CT skills and/or propose methodologies/frameworks or evaluate/compare tools for achieving this goal. In terms of hardware and software, most projects use LEGO products.
\end{abstract}

Resumo. O objetivo deste artigo é apresentar o estado da arte em relação a utilização da robótica educacional como estratégia para o desenvolvimento de habilidades do pensamento computacional (PC) em jovens que estejam na faixa etária do ensino básico. Utilizou-se a metodologia de Revisão Sistemática de Literatura, tanto para planejar e executar a revisão, quanto para apresentar os resultados. Eles mostram que a maioria dos trabalhos objetivam desenvolver habilidades do PC elou propor metodologias ou frameworks ou ainda avaliar/comparar ferramentas para atingir este objetivo. No que diz respeito a hardware e software, a maioria dos projetos utiliza produtos da empresa LEGO.

\section{Introdução}

O Pensamento Computacional (PC) segundo [Wing 2006] é um processo que envolve a "resolução de problemas, a capacidade de projetar sistemas e a compreensão do comportamento humano recorrendo aos conceitos fundamentais da Ciência da Computação". Também é definido pela Computer Science Teachers Association ${ }^{1}$ (CSTA) em [ISTE 2011] como um processo de solução de problemas que inclui (mas não está limitado a) algumas características como: a) formalizar problemas; b) organizar logicamente e analisar dados; c) representar dados por meio de abstrações; d) automatizar soluções por meio de algoritmos; e) identificar, analisar e implementar possíveis soluções; e f) generalizar e converter a solução de um problema para resolver uma ampla variedade de problemas. Essas características devem ser suportadas por um número de disposições

\footnotetext{
* Projeto com chancela da SBC e realizado com o apoio da PREC e PRPPG / UFPel.

${ }^{1}$ CSTA Computational Thinking Task Force. http://www.csta.acm.org/Curriculum/sub/CompThinking.html
} 
VI Congresso Brasileiro de Informática na Educação (CBIE 2017)

Anais do XXVIII Simpósio Brasileiro de Informática na Educação (SBIE 2017)

ou atitudes que são essenciais [ISTE 2011]: a) confiança em lidar com a complexidade; b) persistência para problemas difíceis; c) tolerância com a ambiguidade; d) habilidade para trabalhar um problema do início ao fim; e f) habilidade para comunicar e trabalhar com outros para atingir um objetivo comum.

A robótica educacional [Zilli 2004] tem sido apontada como uma alternativa para o desenvolvimento do PC [Oliveira and Araujo 2016, da Silva et al. 2016, Zanetti and Oliveira 2015]. Isto é, o projeto, desenvolvimento, construção e operação de robôs têm constituído um instrumento sólido e efetivo para o desenvolvimento de habilidades do PC. Conforme reportado em [Neto et al. 2015], diversos trabalhos já demonstram que a robótica constitui uma ferramenta efetiva de ensino, possibilitando o aprendizado de conteúdos [da Costa Barbosa et al. 2015] e a promoção de novas competências.

Este trabalho tem por objetivo apresentar o estado da arte das pesquisas que investigam o desenvolvimento de habilidades/competências do PC em estudantes do ensino básico, utilizando a robótica educacional como estratégia fundamental. Para isto são analisados artigos publicados nos últimos cinco anos que abordam o desenvolvimento do PC, por meio da robótica, com jovens do ensino básico. Adotou-se a Revisão Sistemática de Literatura (RSL) como metodologia para este levantamento. O texto está organizado como segue. A seção 2 descreve, de forma resumida, as etapas que foram desenvolvidas durante a revisão. Na seção 3 são apresentados os resultados, estruturados a partir das questões de pesquisa e na seção 4 são destacadas algumas conclusões.

\section{Metodologia}

Para descrever o "estado da arte" do desenvolvimento do PC por meio da robótica a RSL, apresentada neste artigo, foi baseada em [Kitchenham and Charters 2007] que define os seguintes passos para a elaboração de uma pesquisa: planejamento, formalizado por meio de um protocolo; execução, na qual os dados dos trabalhos são extraídos e analisados; e divulgação dos resultados, onde os dados coletados durante a execução são sistematizados.

Antes de iniciar o processo de construção da RSL, foi realizada uma pesquisa sobre outras revisões já publicadas sobre esse tema. Foram localizadas quatro publicações que tratam, não especificamente do desenvolvimento do PC por meio da robótica, mas da robótica como ferramenta para ensino de programação [Major et al. 2012], robótica nas escolas (educação) [Benitti 2012, Neto et al. 2015] e movimento maker na educação [Papavlasopoulou et al. 2017]. Considerando o fato destas revisões não tratarem, especificamente, sobre PC, prosseguiu-se com a criação de uma nova RSL para conhecer e sintetizar os resultados dos estudos sobre o assunto nos últimos cinco anos (2012 a 2016). Definiu-se como objetivo da pesquisa: "identificar o estado da arte em relação às metodologias e ferramentas que estão sendo utilizadas para desenvolver o PC por meio da robótica educacional". Visando atingir este objetivo foram incluídas no protocolo as questões de pesquisa que são respondidas na seção 3 .

Na etapa seguinte, a string de busca [(“computational thinking") AND (robo* OR lego OR arduino OR raspberry OR "physical computing")] foi submetida às seguintes bases de dados científicas: IEEE, ACM, SpringerLink, ebscohost e Science Direct. A busca retornou 236 artigos. Desses, 24 eram duplicados (apareceram em mais de uma fonte de pesquisa), resultando em 212 artigos. Foram incluídos artigos que reportam a utilização 
VI Congresso Brasileiro de Informática na Educação (CBIE 2017)

Anais do XXVIII Simpósio Brasileiro de Informática na Educação (SBIE 2017)

da robótica educacional no desenvolvimento do PC com jovens do ensino básico (estudos empíricos ou relatos de experiências). Foram excluídos trabalhos que: a) não descreveram uma intervenção com alunos do ensino básico ou com um nível suficiente de detalhamento que permitisse a extração de dados; b) não apresentaram resultados; c) não estavam em inglês; d) estavam duplicados; e) não tratavam de PC e robótica; f) artigos resumidos short papers e g) estudos secundários (RSL por exemplo).

A partir da leitura dos resumos dos trabalhos e aplicando os critérios de inclusão e exclusão, totalizaram 86 artigos a serem analisados. Estes tiveram seus textos examinados na íntegra, considerando-se mais uma vez os critérios de inclusão e exclusão, resultando em 21 estudos (dos quais, 10 publicados em 2016, 5 em 2015, 4 em 2014 e 2 em 2013). A relação completa dos trabalhos incluídos nesta revisão sistemática pode ser visualizada na Tabela 1. O identificador de cada estudo, nomeado de E1 até E21, será utilizado nesta revisão para referenciar os dados e resultados encontrados.

\section{PC por meio da Robótica Educacional}

Nesta seção foram sintetizadas as principais informações sobre as questões de pesquisa consideradas.

QP1 - Quais são os principais objetivos dos estudos e quais são as habilidades/conceitos do PC envolvidos? A Tabela 2 relaciona os objetivos encontrados com os estudos analisados. Em relação aos estudos que foram categorizados em OBJ1, em [E3] foi relatado um projeto piloto que usou robótica (LEGO EV3) e design de jogos para desenvolver e avaliar, por meio de uma rubrica (Computational thinking rubric), a capacidade de formular problemas, de trabalhar com abstração, raciocínio lógico, desenvolvimento de algoritmos e generalização em estudantes do ensino médio. Em [E7 e E9] o objetivo explicitado foi no sentido de gerar interesse na construção de dispositivos e ao mesmo tempo ensinar conceitos básicos de programação e desenvolver habilidades pessoais e interpessoais, como criatividade, resolução de problemas, aprendizado independente e trabalho colaborativo. Em [E10 e E13] as formações tinham como foco a participação de meninas na computação, sendo que em [E13], além do objetivo de capacitar, fica evidente a preocupação com a avaliação da efetividade na utilização de ambientes de programação e dispositivos robóticos para ensinar conceitos de programação (variáveis, repetição, decisão, funções, objetos, entrada/saída, eventos, parâmetros e propriedades). Com foco em crianças mais jovens e utilizando dispositivos de programação tangível (em blocos físicos), o projeto relatado em [E6] procura exercitar alguns conceitos básicos de programação como comandos de sequência e decisão, e depuração.

Em relação às metodologias/frameworks (OBJ2) o estudo [E5] apresenta um método de capacitação em robótica educacional onde são desenvolvidas as seguintes habilidades: decomposição, abstração, generalização, algoritmo e modularização. Em [E21] a metodologia apresentada envolve o uso de 3 plataformas (Kodu, Alice e LEGO) e procura desenvolver conceitos como objetos, variáveis, condicional, paralelismo e repetição. Em [E16] o objetivo é apresentar um método para melhorar a estruturação de atividades de aprendizagem de robótica, evitando sobrecarga cognitiva e distração. Em dois trabalhos [E18 e E20] são apresentados frameworks que envolvem robótica e ciência da computação. O primeiro visando desenvolver habilidades como manipulação de variáveis, comandos de repetição, condicionais e lógica booleana e o segundo, mais 
VI Congresso Brasileiro de Informática na Educação (CBIE 2017)

Anais do XXVIII Simpósio Brasileiro de Informática na Educação (SBIE 2017)

Tabela 1. Relação dos estudos da RSL

ID Referência

E1 BERLAND, M.; WILENSKY, U. Comparing virtual and physical robotics environments for supporting complex systems and computational thinking. Journal of Science Education and Technology, [S.1.], v.24, n.5, p.628-647, 2015.

E2 SULLIVAN, A.; BERS, M. U. Robotics in the early childhood classroom: learning outcomes from an 8-week robotics curriculum in pre-kindergarten through second grade. International Journal of Technology and Design Education, [S.1.], v.26, n.1, p.3-20, 2016.

E3 LEONARD, J. et al. Using Robotics and Game Design to Enhance Children's Self-Efficacy, STEM Attitudes, and Computational Thinking Skills. Journal of Science Education and Technology, [S.1.], v.25, n.6, p.860-876, 2016.

E4 GROUT, V.; HOULDEN, N. Taking computer science and programming into schools: The Glynd wr/BCS Turing project. ProcediaSocial and Behavioral Sciences, [S.1.], v.141, p.680-685, 2014.

E5 ATMATZIDOU, S.; DEMETRIADIS, S. Advancing students' computational thinking skills through educational robotics: A study on age and gender relevant differences. Robotics and Autonomous Systems, [S.1.], v.75, p.661-670, 2016.

E6 BERS, M. U.; FLANNERY, L.; KAZAKOFF, E. R.; SULLIVAN, A. Computational thinking and tinkering: Exploration of an early childhood robotics curriculum. Computers \& Education, [S.1.], v.72, p.145-157, 2014.

E7 THOTA, N.; ESTADIEU, G.; FERRAO, A.; MENG, W. K. Engaging School Students with Tangible Devices: Pilot Project with. NET Gadgeteer. In: LATICE, 2015 INTERNATIONAL CONFERENCE ON, 2015. Anais. . . [S.1.: s.n.], 2015. p.112-119.

E8 CROSS, J. et al. Development of an assessment for measuring middle school student attitudes towards robotics activities. In:FIE, 2016 IEEE, 2016. Anais. . . [S.1.: s.n.], 2016. p.1-8.

E9 ENRIQUEZ, C.; AGUILAR, O. Using robot to motivate computational thinking in high school students. IEEE LAT,[S.1.], v.14, n.11, p.4620-4625, 2016.

E10 ALHUMOUD, S.; AL-KHALIFA, H. S.; AL-RAZGAN, M.; ALFARIES, A. Using app inventor and lego mindstorm nxt in a summer camp to attract high school girls to computing fields. In: EDUCON, 2014 IEEE, 2014. Anais. . . [S.1.: s.n.], 2014. p.173-177.

E11 CROSS, J.; HAMNER, E.; ZITO, L.; NOURBAKHSH, I. Engineering and computational thinking talent in middle school students: a framework for defining and recognizing student affinities. In: FIE, 2016 IEEE, 2016B. Anais. . . [S.1.: s.n.], 2016B. p.1-9

E12 HUGHES, J. Robotic rescue simulation for computing teaching in the UK: A case study. In: GLOBAL ENGINEERING EDUCATION CONFERENCE (EDUCON), 2016 IEEE, 2016. Anais. . . [S.1.: s.n.], 2016. p.1051-1055.

E13 NESIBA, N. et al. Young Women in Computing: Creating a successful and sustainable pipeline. In:FIE, 2015. 32614 2015. IEEE, 2015. Anais. [S.1.: s.n.], 2015. p.1-9.

E14 RUBENSTEIN, M.; CIMINO, B.; NAGPAL, R.; WERFEL, J. AERobot: An affordable one-robot-per-student system for early robotics education. In: ROBOTICS AND AUTOMATION (ICRA), 2015 IEEE INTERNATIONAL CONFERENCE ON, 2015. Anais. .. [S.1.: s.n.], 2015. p.6107-6113.

E15 SHIM, J.; KWON, D.; LEE, W. The Effects of a Robot Game Environment on Computer Programming Education for Elementary School Students. IEEE Transactions on Education, [S.1.], 2016

E16 JIN, K. H.; HAYNIE, K.; KEARNS, G. Teaching Elementary Students Programming in a Physical Computing Classroom. In: ANNUAL CONFERENCE ON INFORMATION TECHNOLOGY EDUCATION, 17., 2016. Proceedings. . . [S.1.: s.n.], 2016. p.85-90.

E17 MERKOURIS, A.; CHORIANOPOULOS, K. Introducing computer programming to children through robotic and wearable devices. In: WORKSHOP IN PRIMARY AND SECONDARY COMPUTING EDUCATION, 2015. Proceedings. . . [S.1.: s.n.], 2015. p.69-72.

E18 CATETÉ, V.; WASSELL, K.; BARNES, T. Use and development of entertainment technologies in after school STEM program. In: ACM TECHNICAL SYMPOSIUM ON COMPUTER SCIENCE EDUCATION, 45., 2014. Proceedings. . . [S.1.: s.n.], 2014. p.163-168.

E19 PINTO-LLORENTE, A. M.; MARTÍN, S. C.; GONZÁLEZ, M. C.; GARCÍA-PEÑALVO, F. J. Developing computational thinking via the visual programming tool: lego education WeDo. In: 4th INTERNATIONAL CONFERENCE ON TECHNOLOGICAL ECOSYSTEMS FOR ENHANCING MULTICULTURALITY, 2016. Proceedings. . . [S.1.: s.n.], 2016. p.45-50.

E20 LARKINS, D. B.; MOORE, J. C.; RUBBO, L. J.; COVINGTON, L. R. Application of the cognitive apprenticeship framework to a middle school robotics camp. In: PROCEEDING OF THE 44TH ACM TECHNICAL SYMPOSIUM ON CS EDUCATION, 2013. Anais. . . [S.1.: s.n.], 2013. p.89-94.

E21 TOURETZKY, D. S. et al. Accelerating K-12 computational thinking using scaffolding, staging, and abstraction. In: PROCEEDING OF THE 44TH ACM TECHNICAL SYMPOSIUM ON COMPUTER SCIENCE EDUCATION, 2013. Anais. . . [S.1.: s.n.], 2013. p.609-614.

focado em robótica, abordando raciocínio lógico, decomposição e resolução de problemas e trabalhando conceitos relacionados à programação (algoritmos sequenciais e programação).

Em relação às ferramentas (OBJ3) em [E1] o objetivo é apresentar e avaliar o software VBOT que permite a manipulação de robôs físicos reais e também robôs simulados em tela. Em [E2] foi avaliado o Kit de robótica KIWI que envolve hardware (o próprio robô) e o software usado para programar o KIWI chamado CHERP. Por sua vez, apresentar um robô de baixo custo chamado AERobot foi o objetivo do estudo [E14] onde 
VI Congresso Brasileiro de Informática na Educação (CBIE 2017)

Anais do XXVIII Simpósio Brasileiro de Informática na Educação (SBIE 2017)

Tabela 2. Relação de objetivos identificados

\begin{tabular}{llll} 
ID & Descrição & Estudos & QTD / (\%) \\
\hline OBJ1 & $\begin{array}{l}\text { Projetos que realizam alguma intervenção com o objetivo de desenvolver habilida- } \\
\text { des do PC }\end{array}$ & $\begin{array}{l}\text { [E3, E4, E6, E7, E9, E10, } \\
\text { E13] }\end{array}$ & $7(33,3)$ \\
OBJ2 & $\begin{array}{l}\text { Projetos que possuem como foco apresentar e/ou avaliar metodologias/frameworks } \\
\text { que envolvam robótica e PC }\end{array}$ & $\begin{array}{l}\text { [E16, E18, E20, E21] } \\
\text { OBJ3 }\end{array}$ & $\begin{array}{l}\text { Projetos que visam apresentar e/ou avaliar a efetividade de ferramentas para o de- } \\
\text { senvolvimento do PC por meio da robótica }\end{array}$ \\
$\begin{array}{l}\text { Projetos que visem apresentar alguma proposta de avaliação do PC em projetos que E14, E15, } \\
\text { OBJ4, E19] } \\
\text { Envolvam robótica educacional }\end{array}$ & $\begin{array}{l}\text { [E8, E11] } \\
\text { OBj,3) }\end{array}$ & $2(9,5)$ \\
\hline
\end{tabular}

foi utilizada uma metáfora de insetos artificiais, com "comportamentos", que junto com os sensores permitia não somente atividades clássicas da robótica (bate-e-volta, seguidor de linha, etc.) e os conceitos básicos de programação e robotização (variáveis, condicionais, repetição e depuração), mas também conceitos mais avançados e explorações filosóficas. O estudo [E17] compara a efetividade das plataformas Scratch, Modkit e Enchanting para desenvolver habilidades de programação (comandos sequenciais, repetição e decisão). Para avaliar a plataforma LEGO Wedo, tanto o software quanto hardware, em [E19] foi investigada a aprendizagem em relação a construção e programação de modelos 3D. Foram destacados os resultados positivos em relação a percepção dos alunos sobre as possibilidades do ambiente no desenvolvimento da capacidade de pensar criativamente, refletir sobre as atividades, resolver problemas de forma lógica e conhecer os resultados de suas decisões. O Algorithmic Bricks (A-Bricks) é avaliado em [E15]. Baseado em programação tangível com pequenos blocos que podem ser conectados na horizontal ou vertical é voltado a crianças dos primeiros anos escolares e permite a aprendizagem de comandos sequenciais, repetição, condicionais, funções e parâmetros.

Por fim, dos projetos com OBJ4, em [E8] é apresentado um instrumento chamado RAAS (robotics activity attitudes scale) que serve para avaliar a percepção (interesse, expectativa, confiança, etc.) em relação a fluência tecnológica dos alunos do ensino médio. Especificamente em relação ao PC, a percepção é medida por meio de perguntas envolvendo raciocínio lógico e problemas complexos. De forma similar, um framework é apresentado em [E11] com o objetivo de reconhecer talentos de alunos nas áreas de PC (resolução de problemas, abstração e pensamento algorítmico) e engenharia.

QP2 - Quais são as metodologias que estão sendo utilizadas para desenvolvimento do $\mathrm{PC}$ em crianças e jovens por meio da robótica? Os seguintes métodos foram adotados para a promoção do PC por meio da robótica educacional: M1 intervenções desenvolvidas por meio de roteiros e tarefas em atividades práticas, colaborativas e divertidas. Em geral essa abordagem se aproxima com a ideia construcionista [Papert 1980]; M2 - intervenções baseadas na teoria da aprendizagem cognitiva [Collins et al. 1991]; M3 - aprendizagem baseada em projetos/problemas (PBL) [Strobel and Van Barneveld 2009, Thomas 2000]; M4 - intervenção estruturada a partir dos conceitos de desenho instrucional Activity-Media Design que visa a diminuição da sobrecarga cognitiva em atividades de robótica [Sweller 1994]; M5 - intervenção baseada em um modelo de três estágios com transição entre ambientes de programação (KODU, Alice, NXT-G) e diminuição na estruturas de assistência/suporte [Marghitu et al. 2010]; M6 - competição de robótica em taster days; M7 - combinação de robótica educacional e desenvolvimento de jogos (game design). A Figura 1 correlaciona os métodos com os 
VI Congresso Brasileiro de Informática na Educação (CBIE 2017)

Anais do XXVIII Simpósio Brasileiro de Informática na Educação (SBIE 2017)

objetivos (QP1) e distribui os estudos.

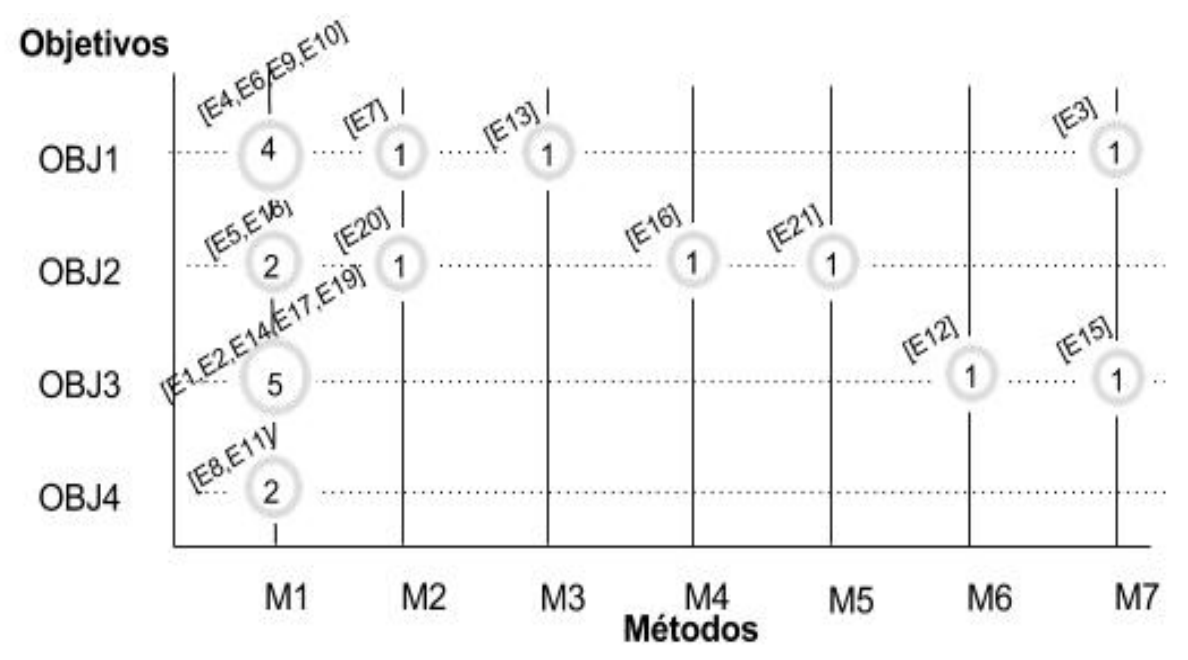

Figura 1. Objetivos e respectivas metodologias

Foi possível identificar que projetos com objetivos diferentes acabam adotando um método bastante similar para trabalhar a temática PC e robótica educacional. O método identificado por M1, onde os aprendizes trabalham em grupos seguindo roteiros e/ou realizando tarefas/desafios, normalmente lúdicos, foi identificado em 13 dos 21 estudos desta RSL. Em geral é dada alguma instrução no início do curso em relação ao funcionamento dos robôs e também é apresentado um ambiente de programação.

Em [E1], antes de iniciar às atividades, os pesquisadores se reuniram com o professor regular da turma para apresentar a plataforma. As atividades foram ministradas pelos pesquisadores em conjunto com o professor. O pesquisador ensinou a sintaxe VBOT para os alunos no início dos encontros, utilizando um conteúdo programático predeterminado. Foram, ao todo, 5 encontros com desafios a serem realizados pelos alunos. De forma bem similar, em [E2 e E6] as capacitações começaram com uma introdução a robótica e programação baseada na plataforma TangibleK e linguagem CHERP. As aulas foram ministradas uma vez por semana e duraram aproximadamente $1 \mathrm{~h}$. Foram desenvolvidos conteúdos que tratavam do funcionamento dos dispositivos, formato da programação tangível, sensores de distância, som e luz e motores. O projeto final, em [E2] envolveu um mapa do bairro onde as crianças foram desafiadas a programarem seus robôs parando em determinados locais e realizando diferentes ações. Já em [E6] um projeto interdisciplinar que convida crianças a aplicar o conhecimento adquirido a um tema ou contexto específico. O professor decide sobre um tema extraído de outras disciplinas estudadas durante o ano e cada criança escolhe um desafio dentro desse tema.

Em [E4] as sessões começaram com uma introdução ao hardware e software a serem usados, seguido de uma demonstração simples dos robôs e placas. O primeiro desafio, mais simples, trata de estender a função de um dos exemplos. Os exercícios tornam-se mais desafiadores e as crianças passam a resolver problemas mais complexos que os primeiros. Durante dois ou três meses os alunos trabalharam remotamente, com desafios que eram propostos no site do projeto e com feedback remoto. Depois desta etapa um novo encontro presencial foi realizado onde os robôs e placas voltaram para a sala de aula e as soluções dos alunos foram implementadas e avaliadas. O progresso foi discutido 
VI Congresso Brasileiro de Informática na Educação (CBIE 2017)

Anais do XXVIII Simpósio Brasileiro de Informática na Educação (SBIE 2017)

com as crianças e seus professores e também completaram um questionário de feedback. Em [E5] a abordagem didática é similar ao que foi relatado nos estudos anteriores, com destaque para o suporte (scaffold) aos estudantes que é diminuído gradualmente após a 5a sessão (de um total de 11).

Um acampamento de verão onde ocorreu formação em robótica é relatado em [E10]. No primeiro dia os alunos tiveram a oportunidade de experimentar diferentes sensores, utilizar funções e mini-programas. Nos demais dias os alunos foram orientados a construir um robô a partir do zero usando um manual, a discutir as ideias do projeto final e a construir protótipos. Com diversas montagens e desmontagens os estudantes adquiriram destreza e também trabalharam a criatividade. Nos últimos dias, os alunos foram convidados a construir um robô que deveria executar três ações diferentes utilizando pelo menos dois sensores e, finalizando, realizaram a apresentação aos juízes para a seleção do melhor trabalho. Ao final de cada dia foi realizada uma rápida sessão de encerramento para feedback e desmontagem dos robôs. Similarmente em [E18] é relatado um amplo programa de extensão onde, no módulo de robótica, os alunos criam e desenvolvem programas para robôs usando LEGO Mindstorms. Eles primeiro usam instruções guiadas para aprender a construir um robô e programá-lo para fazer tarefas simples. Depois formam equipes e criam seus próprios projetos para competir com outras equipes.

Em [E17] é realizada uma comparação entre 3 ambientes, onde 36 alunos realizaram algumas tarefas a partir de um material instrucional que objetivava representar os mesmos conceitos computacionais para cada plataforma alvo. As atividades se concentraram em 3 conceitos da computação: comandos sequenciais, repetição e decisão. Também com a intenção de avaliar a ferramenta LEGO Wedo, em [E19] os alunos foram divididos em grupos de 3 alunos. Um com nível básico de conhecimento, outro intermediário e um com conhecimento avançado. Cada equipe teve que construir e programar dois modelos 3D na ferramenta: Dancing Birds e Smart Spinner. No Dancing Birds a tarefa consistia em construir e programar duas aves mecânicas motorizadas que produzissem sons para dançar a partir de um sistema de roda e correia. No Smart Spinner era necessário construir e programar um mecanismo giratório motorizado com sensor de movimento para desligar o motor quando um pião é lançado. Diversos conteúdos interdisciplinares (ciências, tecnologia, engenharia e matemática) são explorados nas atividades.

Em [E8 e E11] o foco está na avaliação do projeto Art \& Bots, onde os alunos combinam dispositivos robóticos, programação de computadores e artesanato para criar esculturas robóticas ligadas a objetivos curriculares ou ligados a interesses criativos, como música, arte e narração de histórias. Em função do foco em avaliação, os estudos não abordam muitos detalhes da metodologia das atividades que visam desenvolver habilidades do PC. Similarmente, em [E14], onde é apresentado um kit de robótica chamado AERobot, algumas fotos levam a inferir que os participantes trabalharam em um formato mais livre e com incentivo a criatividade a partir de uma combinação de robótica com outros materiais, como papelão e pintura para a personalização dos dispositivos. Também em [E9] não foi possível determinar com exatidão como foram realizadas as atividades ou desafios, porém no artigo os autores afirmam que "o trabalho desenvolvido é baseado no uso de um robô como uma ferramenta de ensino tendo em conta as teorias construtivistas, enfatizando o desenvolvimento da criatividade e a colaboração".

Em relação ao método M2, dois estudos [E7 e E20] ancoram a metodologia na 
aprendizagem cognitiva (AC) [Collins et al. 1991] utilizando a seguinte abordagem: a) modeling - demonstração pelo professor; b) coaching - professor observa os alunos e fornece ajuda quando necessário; c) scaffolding - o professor auxilia em tarefas que estão além das habilidades do aluno. A assistência é lentamente retirada (fading) na medida em que o aluno consegue desenvolver atividades por conta própria; d) articulação - exige que os alunos pensem sobre suas próprias ações e as expliquem aos outros; e) reflexão - alunos refletem sobre seu próprio desempenho na solução de um problema; e f) exploração - investigação de novos métodos, estratégias e teste de novas hipóteses. Cada um dos métodos tem uma concepção de aprendizagem, um formato que define como uma determinada "aula" deva ser executada, com mais ou menos intervenção do professor.

O método M3 está relacionado com a aprendizagem baseada em projetos ou problemas (PBL) que foi utilizado no estudo [E13] onde é descrita uma iniciativa para ampliar a participação de mulheres na computação. O método M4, que trata de design instrucional tem como preocupação a sobrecarga cognitiva de intervenções que envolvam robótica, ou seja, a metodologia proposta em [E16], Activity-Media Design, tem como etapa fundamental analisar o uso de mídias em cada atividade para identificar e redesenhar aquelas que utilizam um número excessivo de informações verbais ou visuais. Em relação ao método M5, o projeto relatado em [E21] apresenta um modelo de três estágios de formação em programação e robótica começando com um ambiente simples, chamado $\mathrm{Kodu}^{2}$ e progredindo para estruturas mais desafiadoras (Alice ${ }^{3}$ e Lego NXT-G). O método M6 se baseia na ideia de competição. A intervenção relatada em [E12], que visava avaliar o software CoSpace Rescue, foi iniciada com uma série de pequenas demonstrações e explicações sobre o software e depois os alunos realizaram tarefas. Posteriormente foram realizadas três torneios entre duplas.

O método M7 relaciona estudos que utilizam jogos e robótica. Em [E3], além de roteiros e atividades de robótica, o projeto também utiliza, para desenvolver habilidades do PC, o desenvolvimento de jogos com os softwares AgentSheets e AgentCubes [Repenning et al. 2010]. Em [E15] a metodologia do projeto se baseia na filosofia "Edutainment" que "combina conceitos de educação e entretenimento para que os alunos se envolvam em auto-aprendizagem enquanto jogam, resultando em um aumento na qualidade da educação" [Bourgonjon et al. 2013] efetivada por meio de jogos com programação tangível com o dispositivo A-Bricks. No artigo os autores exemplificam os jogos "Menor rota", "Jogo de Corrida" como desafios a serem resolvidos por meio da programação tangível e utilizando os conceitos básicos de programação (sequência, condição, repetição, funções e parametrização). Os alunos participaram de $8 \mathrm{~h}$ de atividades em uma aula experimental e foram submetidos a uma avaliação que mediu a usabilidade, entretenimento, atitudes de programação e compreensão de conceitos de programação. Essas competições, de curta duração e amigáveis, cativaram os alunos e melhoraram o foco nas atividades.

QP3 - Quais são as ferramentas que estão sendo utilizadas para desenvolvimento do PC em jovens por meio da robótica? Em relação aos de kits de robótica, dos 21 estudos, em 13 foram utilizados kits da empresa LEGO. Dois projetos trabalham com ambientes simulados (VBot Virtual [E1] e CoSpace Rescue [E12]) e em outros dois não

\footnotetext{
${ }^{2}$ Disponível em: $<$ http://www.kodugamelab.com/About $>$. Acesso em:18 jun 2017

${ }^{3}$ Disponível em: $<$ http://www.alice.org $>$. Acesso em: 18 jun 2017
} 
VI Congresso Brasileiro de Informática na Educação (CBIE 2017)

Anais do XXVIII Simpósio Brasileiro de Informática na Educação (SBIE 2017)

é citado qualquer tipo de kit [E8 e E11]. Dos que utilizam kits LEGO, o modelo NXT foi o mais utilizado, com 8 estudos, seguido pelos modelos EV3 e WEDO com um estudo cada. Em três projetos [E6, E16, E18] não foi possível determinar, especificamente, qual kit da LEGO foi utilizado. Outros dispositivos citados foram: Arduino Lilypad [E13 e E17], Picoboard/Picocrickets [E4 e E13], KIWI robotics kit [E2], Raspberry PI [E4], Vbot [E1], Microsoft .NET Gadgeteer [E7], A-Bricks [E15] e AERobot kit [E14].

Em relação ao software, o ambiente gráfico da empresa LEGO é o mais utilizado, sendo citado em 8 projetos. Diversos ambientes foram citados nas pesquisas, porém não foi possível estabelecer, pelo menos no escopo desta revisão, uma outra tendência ou efetiva utilização de determinado(s) ambiente(s) de programação para dispositivos robóticos.

\section{Conclusões}

O presente trabalho procurou investigar o estado da arte no que diz respeito a utilização da robótica como estratégia para desenvolvimento de habilidades e competências do PC. Em relação aos objetivos, observou-se que quatro diferentes categorias de objetivos estavam presentes nos estudos (Tabela 2), divididos, quase que igualmente, entre OBJ1, OBJ2 e OBJ3. Apenas dois estudos tiveram como foco a avaliação (OBJ4). No que diz respeito aos métodos ou metodologia das intervenções, a maioria dos projetos trabalha com roteiros e tarefas em atividades práticas, colaborativas (em grupos) e divertidas/lúdicas (M1).

Em relação aos kits de robótica utilizados nos estudos que fazem parte desta RSL, é possível observar claramente a liderança dos kits da empresa LEGO. Sendo assim, ainda não é tão frequente a utilização de placas controladoras do tipo hardware livre, como Arduino, ou equipamentos do tipo mini-pc como Raspberry PI. Essa constatação vai ao encontro de [Benitti 2012], uma revisão sistemática sobre robótica nas escolas, que constatou um pequeno número de pesquisas envolvendo kits de baixo custo, relatando que 90\% dos projetos utilizavam LEGO.

A temática do desenvolvimento de competências e habilidades usando a robótica como estratégia ainda é bastante recente. De forma mais abrangente, o próprio conceito de PC, definido em 2006 [Wing 2006] também é novo e carece de mais pesquisas para embasar intervenções e permitir a avaliação de sua efetividade enquanto "desenvolvedor" de habilidades e competências importantes para a vida de jovens. Outro aspecto observado nesta RSL foi a pouca atenção dada a interdisciplinaridade. Apenas quatro estudos [E6, E19, E20 e E21], dos 21 que fazem parte desta revisão, possuem, explicitamente, alguma referência a conteúdos ou atividades interdisciplinares.

Portanto, o PC, tendo a robótica como estratégia, é um tema onde diversas questões estão em aberto, principalmente metodológicas, no sentido de guiar a realização de intervenções que visem desenvolver habilidades do PC. Também existem questões de cunho prático pois, para uma ampla disseminação, é preciso investimento em capacitação de profissionais e aquisição de equipamentos (dispositivos robóticos). Enfim, pesquisas devem ser realizadas para estabelecer estratégias de intervenções realmente efetivas para a melhoria da capacidade cognitiva de crianças e jovens.

\section{Referências}

Benitti, F. B. V. (2012). Exploring the educational potential of robotics in schools: A systematic review. Computers \& Education, 58(3):978-988. 
VI Congresso Brasileiro de Informática na Educação (CBIE 2017)

Anais do XXVIII Simpósio Brasileiro de Informática na Educação (SBIE 2017)

Bourgonjon, J. et al. (2013). Acceptance of game-based learning by secondary school teachers. Computers \& Education, 67:21-35.

Collins, A., Brown, J. S., and Holum, A. (1991). Cognitive apprenticeship: Making thinking visible. American educator, 15(3):6-11.

da Costa Barbosa, F. et al. (2015). Robótica educacional em prol do ensino de matemática. In Anais do Workshop de Informática na Escola, volume 21, page 271.

da Silva, D. P. et al. (2016). Aplicação de robótica na educação de forma gradual para o estímulo do pensamento computacional. In Anais dos Workshops do CBIE 2016, pages 1188-1197.

ISTE, C. (2011). Computational thinking in k-12 education leadership toolkit.

Kitchenham, B. A. and Charters, S. (2007). Guidelines for performing systematic literature reviews in software engineering.

Major, L., Kyriacou, T., and Brereton, O. P. (2012). Systematic literature review: Teaching novices programming using robots. IET software, 6(6):502-513.

Marghitu, D. et al. (2010). Auburn university k-12 inclusive stem outreach programs. In SDPS2010 Conference, pages 6-9.

Neto, R. P. B., Santana, A. M., Rocha, D. P., and Souza, A. (2015). Robótica na educação: Uma revisão sistemática dos últimos 10 anos. In Anais do SBIE 2015, pages 386-395.

Oliveira, E. and Araujo, A. L. (2016). Pensamento computacional e robótica: Um estudo sobre habilidades desenvolvidas em oficinas de robótica educacional. In Anais do SBIE 2016, pages 530-539.

Papavlasopoulou, S. et al. (2017). Empirical studies on the maker movement, a promising approach to learning: A literature review. Entertainment Computing, 18:57-78.

Papert, S. (1980). Mindstorms:Children,computers, and powerful ideas. Basic Books, Inc.

Repenning, A., Webb, D., and Ioannidou, A. (2010). Scalable game design and the development of a checklist for getting computational thinking into public schools. In Proceedings of the 41st ACM technical symposium on Computer science education, pages 265-269. ACM.

Strobel, J. and Van Barneveld, A. (2009). When is pbl more effective? a meta-synthesis of meta-analyses comparing pbl to conventional classrooms. Interdisciplinary Journal of Problem-based Learning, 3(1):4.

Sweller, J. (1994). Cognitive load theory, learning difficulty, and instructional design. Learning and instruction, 4(4):295-312.

Thomas, J. W. (2000). A review of research on project-based learning.

Wing, J. M. (2006). Computational thinking. Communications of the ACM, 49(3):33-35.

Zanetti, H. and Oliveira, C. (2015). Práticas de ensino de programação de computadores com robótica pedagógica e aplicação de pensamento computacional. In Anais dos Workshops do CBIE, pages 1236-1245.

Zilli, S. d. R. (2004). A robótica educacional no ensino fundamental: perspectivas e prática. Dissertação de mestrado, PPGEP/UFSC, Florianópolis/SC. 